\title{
La situación de los niños, niñas y adolescentes migrantes en la Argentina, y la respuesta del Estado previa y durante la emergencia sanitaria COVID-19
}

\author{
The Situation of Migrant Children and Adolescents in \\ Argentina, and the Response of the State Prior to and During \\ the COVID-19 Health Emergency
}

MARIANA AHUALLI ${ }^{1}$

Resumen: La emergencia sanitaria COVID-19 ha presentado desafíos sin precedentes al mundo entero y ha exacerbado desigualdades preexistentes en las sociedades. Los Estados, para dar respuesta, han diseñado diversas políticas económicas y sociales que intentan paliar las consecuencias negativas sobre las familias. Este trabajo muestra cómo, en la Argentina, el desarrollo de nuevas políticas, en lugar de subsanar deficiencias del pasado, profundiza las desigualdades en el acceso a derechos entre la población, excluyendo a niñas, niños y adolescentes sobre la base de su condición migratoria. Estas políticas contradicen los marcos normativos internacionales y regionales, así como los nacionales, y su gravedad se ve acentuada por los índices de pobreza y las vulneraciones de derechos que presenta este grupo poblacional. Los niños y niñas migrantes se encuentran en

\footnotetext{
${ }^{1}$ Licenciada en Comunicación, cuenta con una Diplomatura en Migrantes y Protección de Refugiados. Trabaja hace más de 10 años en Naciones Unidas, especializada en temáticas de derechos humanos y sector privado, con particular énfasis en derechos de la infancia y personas con necesidades de protección internacional. Coordinó numerosas publicaciones sobre la temática.
} 
una situación de doble vulnerabilidad, por su condición de niños y su condición de migrantes, lo que resulta en niveles de pobreza más altos.

Palabras claves: Emergencia sanitaria COVID-19, niñez migrante y refugiada, pobreza, derechos de las personas migrantes y refugiadas, políticas sociales, Argentina.

Abstract: The COVID-19 sanitary emergency has presented unprecedented challenges to the entire world and exacerbated preexisting inequalities in societies. In order to respond, the States have designed various economic and social policies that try to alleviate the negative consequences on families. This article shows how, in Argentina, the development of new policies, instead of correcting deficiencies of the past, deepens inequalities in access to rights among the population, excluding children on the basis of their migratory status. These policies contradict international and regional regulatory frameworks, as well as national ones, and their severity is accentuated by the poverty rates and rights violations that this population group endures. Migrant children are in a situation of double vulnerability, due to their status as children and their status as migrants, which results in even higher levels of poverty.

Keywords: COVID-19 sanitary emergency, migrant and refugee children, poverty, rights of migrants and refugees, social policies, Argentina.

Recibido: 18.8.2020

Aceptado: 27.11.2020

\section{Sumario}

\section{Introducción}

2. Marcos normativos: niñez y migración

3. Niñez, migración y pobreza

4. Políticas de protección social para la niñez migrante 


\section{La respuesta del Estado frente a la emergencia sanitaria COVID- 19}

\section{Reflexiones finales}

\section{Introducción}

Este trabajo tiene por objetivo analizar los desafíos que existen en el cumplimiento de los derechos de niñas, niños y adolescentes migrantes en la Argentina, a partir de estadísticas sobre su situación y una recopilación de las políticas públicas y sus criterios de inclusión y exclusión. Para ello, se desarrollarán tres secciones. En la primera, se presentará el marco normativo correspondiente a nivel internacional, interamericano, regional y nacional. En segundo lugar, se definirá la población sobre la que se hará foco y se presentarán sus condiciones de vida a partir de estadísticas de pobreza desde el punto de vista del hogar, como así también, de las personas. En la tercera y última sección, se analizará el acceso de los niños, niñas y adolescentes migrantes o hijos e hijas de migrantes, a las principales políticas públicas dirigidas a la infancia, diseñadas por el Estado argentino, tanto previo a la emergencia sanitaria COVID-19 como durante la pandemia. Esta distinción se considera de interés, ya que el gobierno ha diseñado nuevos instrumentos para dar respuesta a la crisis, los cuales podrían servir para subsanar desafíos en las políticas preexistentes o, por el contrario, profundizar desigualdades entre la población, basadas en la condición migratoria. La metodología utilizada se basa en el análisis de informes de organismos internacionales y regionales, análisis de normativa y procesamiento de fuentes primarias de información.

El punto de partida son los niños, niñas y adolescentes, debido a que se encuentran en una situación de doble vulnerabilidad, dada por la combinación de su condición de niños y su condición de migrantes. Es por esto que, a lo largo de los años, se ha desarrollado una multiplicidad de instrumentos internacionales, regionales $\mathrm{y}$ 
nacionales orientados a protegerlos, considerando su situación particular, ampliando los estándares de protección y sumando derechos, principios e interpretaciones crecientemente garantistas. Estos cambios se han visto plasmados en avances normativos y jurisprudenciales en el sistema universal, en el sistema interamericano, en espacios subregionales —como el del MERCOSUR - y en legislaciones y políticas nacionales. El marco normativo que regula las migraciones en la Argentina es un modelo para la región y el mundo, y equipara sus derechos con los de la población nacida en su territorio. Sin embargo, es menester evaluar las condiciones materiales de vida y el acceso efectivo a derechos, para identificar dónde están los desafíos pendientes.

Para identificar el foco del trabajo, es necesario realizar dos aclaraciones preliminares, vinculadas al tipo de migración así como al conjunto de niñas, niños y adolescentes. Si bien la OIM define al migrante como una persona que se aleja de su lugar de residencia habitual, ya sea dentro de un mismo país o atravesando una frontera internacional, este trabajo se circunscribe a migrantes internacionales. Por otra parte, las niñas y niños afectados por la migración internacional son un grupo que incluye una diversidad de situaciones: aquéllos que se han trasladado con uno o ambos progenitores; aquéllos que han migrado no acompañados ${ }^{2}$; quienes lo han hecho separados de sus padres, madres, o tutores legales, pero no de otros parientes; hijas e hijos de migrantes nacidos con posterioridad a la migración de sus progenitores ${ }^{3}$, y niñas y niños que permanecen en el país de origen cuando migran sus padres. Estos grupos se pueden superponer con el de aquellos niños migrantes que se encuentran en situación irregular en la región (IPPDH, OIM, 2016). A lo largo del trabajo se intentará hacer referencia a los subgrupos,

\footnotetext{
${ }^{2}$ Son quienes se encuentran fuera de su país de origen, separados de ambos padres y otros parientes, y no están al cuidado de un adulto al que, por ley o costumbre, incumbe esa responsabilidad.

${ }^{3}$ Ellas y ellos adquieren la nacionalidad del país de destino en virtud del principio ius soli, que rige en prácticamente toda la región.
} 
entendiendo que la información disponible varía en los distintos casos.

\section{Marcos normativos: niñez y migración}

Como se ha mencionado anteriormente, los niños, niñas y adolescentes se encuentran en una situación de especial vulnerabilidad, por lo que requieren de protección especial. "Entre las múltiples situaciones que agravan la condición de vulnerabilidad de niños y niñas se cuenta la condición de migrantes. Los migrantes son un grupo en situación de vulnerabilidad, debido a situaciones de jure (desigualdades entre nacionales y extranjeros en las leyes) y de facto (desigualdades estructurales). Esta situación conduce a variadas formas de desigualdad en el acceso a los recursos públicos administrados por los Estados" (IPPDH, OIM, 2016, p 13).

La Convención sobre los Derechos del Niño (CDN) estipula obligaciones a los Estados parte para proteger los derechos de niñas y niños bajo su jurisdicción y, uno de sus principios fundamentales, es la no discriminación, por lo que la condición migratoria es irrelevante a la hora de dar cumplimiento a la CDN. En términos normativos, es necesario mencionar dos instrumentos adicionales. Por un lado, la Observación General Nro. 6, sobre el Trato de los menores no acompañados y separados de su familia fuera de su país de origen, elaborada en el 2005 y, por otro, la Convención internacional sobre la protección de los derechos de todos los trabajadores migratorios y de sus familiares (CTM), que entró en vigor en 2003 y que la Argentina ha ratificado. El interés de esta Convención radica en que amplía el concepto de trabajador migrante a aquél que se prepara para abandonar el país, pero aún no lo ha hecho. En ese sentido, es posible interpretar que también incorpora dentro del término migrante a aquellas niñas y niños que no han abandonado su país, pero cuyos padres y madres sí lo han hecho, por lo que tienen planes de reunirse con ellos en el futuro (IPPDH, OIM, 2016, p. 17). Asimismo, la CTM 
incluye a las hijas e hijos de migrantes, sean ellos mismos migrantes o no.

En cuanto al sistema interamericano de derechos humanos, si bien no existe un tratado específico sobre los derechos de niños y niñas migrantes, "esta protección surge del artículo 7 de la Declaración americana de los derechos y deberes del hombre y del artículo 19 de la Convención americana sobre los derechos humanos. Esas garantías han motivado la producción de una amplia cantidad de informes por parte de Comisión Interamericana de Derechos Humanos (CIDH), así como una vasta jurisprudencia por parte de la Corte Interamericana de Derechos Humano (Corte IDH), e incluso la emisión de una Opinión Consultiva específica sobre el tema" (IPPDH, OIM, 2016, p. 23). La Opinión Consultiva 21/2014, sobre los derechos y garantías de niñas y niños en el contexto de la migración y/o en necesidad de protección internacional, es una herramienta vinculante para todos los Estados parte. En ella se estipula: la prohibición de la privación de la libertad por condición migratoria irregular, el principio de no devolución, el derecho a la vida en familia, el régimen jurídico de infancia por sobre el migratorio y la prohibición de la separación familiar por expulsión de uno o ambos progenitores por infracciones migratorias de carácter administrativo.

En el ámbito local, tanto la Ley 25.871 de Migraciones, que entró en vigencia en 2004, como la Ley 26.061 de Protección Integral de los Derechos de Niñas, Niños y Adolescentes, promulgada en 2005, contribuyen a crear una nueva perspectiva basada en el enfoque de derechos, tanto en materia de niñez como de migraciones. Asimismo, la Ley de Migraciones ofrece un marco jurídico protector de la infancia migrante, vinculado al ingreso, la residencia, y la separación familiar. El criterio de radicación por familia facilita el acceso a la residencia tanto de niñas y niños migrantes, como de sus progenitores (Art. 22 de la Ley y del Reglamento), y la garantía del derecho de reunificación familiar previene decisiones como las de expulsión. Por último, a través de la reglamentación se establece que, para solicitar la residencia de una persona migrante menor de 18 años, basta la 
presentación y autorización de uno de sus progenitores o tutor (Art. 8, inciso d).

En este sentido, es necesario mencionar los avances y retrocesos que acontecieron en los últimos años. Por un lado, debido a la crisis humanitaria que atraviesa Venezuela, el Gobierno argentino estableció, a través de la Disposición 520/2018 de la Dirección Nacional de Migraciones, el ingreso de menores de 9 años que no cuenten con cédula de identidad, mediante la presentación de la partida de nacimiento, de manera excepcional. Esta disposición busca facilitar el ingreso de niñas y niños venezolanos con sus familias. Por otro, cabe mencionar el Decreto de Necesidad y Urgencia 70/2017, cuya constitucionalidad está pendiente de resolución en la Corte Suprema. Este habilita expulsiones que violan el derecho a la defensa y el acceso a la justicia, y su aplicación produce la separación de familias.

\section{Migración, niñez y pobreza}

Según la Encuesta Permanente de Hogares, para el 2018 el 5,4\% de la población urbana en la Argentina era migrante, mientras que el $10,4 \%$ de los hogares contaba con al menos un migrante entre sus miembros (OIM, CNCPS, 2019, p. 29). El peso de los niños, niñas y adolescentes en la población migrante es de 7,4\%, mientras que este número asciende a $29,5 \%$ en la población no migrante. Entre los migrantes, se observa una mayor proporción de hogares con jefatura femenina ( $45 \%$, en comparación con $41 \%$ para los no migrantes).

Dado que "son las necesidades de consumo y los ingresos del hogar las que determinan si las personas que viven en él son pobres o no" (OIM, CNCPS, 2019, p. 20), y esto es principalmente cierto para los niños, niñas, y adolescentes, se considerará, en primer lugar, la pobreza monetaria en función del porcentaje de hogares que son pobres, para luego presentar reflexiones sobre las estadísticas individuales. En ese sentido, la pobreza de los hogares donde al 
menos una persona es migrante es de $25,6 \%$, mientras que el número es de $20,8 \%$ en hogares sin migrantes. Esta brecha se acentúa si se analiza la indigencia $(6,3 \%$ contra $4,1 \%)$. Ahora bien, si desagregamos estos números, comparando hogares con jefas o jefes de hogar y cónyuges migrantes con aquéllos de núcleo mixto, se identifican grandes diferencias. En los hogares con jefe o jefa y cónyuge migrante, la pobreza alcanza $34,6 \%$ y la indigencia $8,9 \%$ (más del doble que los hogares sin migrantes). Aquellos hogares donde uno o una de los miembros (jefe, jefa o cónyuge) es migrante, es decir, de núcleo mixto, la incidencia de la pobreza disminuye once puntos $(23,8 \%)$.

Por otra parte, la pobreza se da con mayor frecuencia en los hogares migrantes donde hay una sola persona jefa de hogar, sin cónyuge y con menores de edad a cargo, que representan el 15,7\% del total de los hogares migrantes. En este subgrupo de hogares la pobreza alcanza $36,7 \%$ y, si se excluye a los jefes varones, aumenta a $40 \%$. Desde una perspectiva de género, vale resaltar que “ $[\mathrm{m}]$ ientras que el $83 \%$ de los/as jefes/as de estos hogares son mujeres, la femenización de la jefatura es más alta cuando los hogares de este tipo son pobres, y alcanza el 90\%” (OIM, CNCPS, 2019, p. 42).

Por último, es importante aclarar que, si se desagregan los datos según la cantidad de miembros en el hogar, los hogares con algún miembro migrante son menos numerosos, por lo que no es esta una variable que incida en la pobreza. “[L] as causas del fenómeno de insuficiencia de ingresos deriva de la desigual inserción laboral en el mercado de trabajo" (OIM, CNCPS, 2019, p.39).

Para complementar el análisis anterior, se hará foco en la pobreza de las personas. En primer lugar, el segmento etario con mayores tasas de pobreza e indigencia entre migrantes son los niños, niñas y adolescentes, con valores de $50,3 \%$ y 16,4\%, respectivamente. Entre nativos, estos valores descienden a $44.3 \%$ y $9,4 \%$, respectivamente. En el interior de este gran grupo existen diferencias significativas. La situación más acuciante la sufren los menores de 18 años argentinos, pertenecientes a hogares donde el jefe o la jefa de 
hogar es migrante y no tiene cónyuge $(60,6 \%$ de pobreza), y donde ambos padres o madres son migrantes $(58,4 \%)$, así como los niños, niñas y adolescentes migrantes (50,3\%). Con porcentajes más bajos de pobreza, aparecen los menores de 18 años argentinos, con uno de los padres migrantes $(43,7 \%)$, que tienen un nivel de pobreza equivalente al de aquellos con ambos padres argentinos.

En el caso de la indigencia, los porcentajes ascienden a $16,4 \%$ para los niños, niñas y adolescentes migrantes, y a $15,1 \%$ para aquellos con ambos padres migrantes, mientras que, para el resto de la infancia, el porcentaje ronda el 9,4\%. "En definitiva, los hogares con jefe/a de hogar migrante, solo/a y con menores a cargo son la tipología de hogar donde tienen lugar las tasas de pobreza infantil más altas. En estos hogares se pone en juego una serie de factores que dejan de manifiesto la vulnerabilidad a la que está expuesta la jefa de hogar, en su doble condición de migrante y mujer" (OIM, CNCPS, 2019, p. 50). Si bien no se ahondará en la pobreza multidimensional, vale aclarar que entre la población migrante se observan privaciones en cuanto a las condiciones habitacionales, cobertura de salud y asistencia escolar de niños y niñas.

Por último, una de las características salientes de las personas migrantes pobres es que, en su mayoría, tienen una tasa de desempleo menor que las argentinas, por lo que su condición de pobreza no es consecuencia de la ausencia de ingresos, sino de una mayor precariedad en la inserción laboral y una insuficiencia de los ingresos para cubrir la canasta básica del hogar.

\section{Políticas de protección social para la niñez migrante}

Las mayores tasas de pobreza e indigencia de los niños, niñas y adolescentes nacidos en el exterior y de argentinos y argentinas cuyos progenitores son migrantes, requieren un análisis sobre las políticas de protección social dirigidas a la infancia, en particular, la 
transferencia de ingresos, que se destaca como una de las políticas centrales de reducción de la pobreza en la Argentina. La evidencia empírica de distintos países muestra que las transferencias de ingresos tienen impactos positivos en la reducción de la pobreza y que "[l]as prestaciones por hijos que son universales o tienen una amplia cobertura de población también pueden tener un impacto claro y significativo en la desigualdad de ingresos" (ODI, UNICEF, 2020, p. 83). En particular, la evidencia en la Argentina muestra que las transferencias monetarias resultan instrumentos eficaces para disminuir el riesgo de pobreza por ingresos y, más significativamente, la pobreza extrema. Los efectos son mayores en hogares cuyo jefe o jefa es un trabajador por cuenta propia o asalariado informal (UNICEF, UNGS, IELDE, 2018), casos muy frecuentes entre la población migrante. La Asignación Universal por Hijo (AUH) representa un pilar fundamental de la protección social de la niñez y prevención de la pobreza, por lo que es necesaria una discusión sobre su configuración, alcance y cobertura.

Una de las principales limitaciones de la AUH es la exclusión de niñas, niños y adolescentes migrantes que no tengan residencia de al menos tres años en la Argentina, así como de aquéllos cuyos padres son extranjeros y no tienen esos años de residencia en el país. Esto se establece en el marco del Decreto 1602/09, cuya redacción es ambigua y no clarifica si la exigencia de 3 años de residencia se refiere a los niños o a sus padres. Esta condición entra en contradicción con los estándares de derechos humanos, ya que el principio de no discriminación incluye cualquier condición del niño o de sus padres o representantes legales (CDN, Art. 2; Ley 26.061, Art. 1 y 28). La inequidad se acentúa si se considera que los hijos de residentes legales con empleo formal perciben la asignación por familiar hijo prevista por la Ley 24.714, sin ningún requisito de residencia.

La Resolución 393/09 de ANSES restringe aún más lo establecido por el decreto, ya que, en su Art. 5, exige que tanto el titular como el niño, niña o adolescente tengan una residencia legal de 3 años, generando una doble discriminación: tanto por la nacionalidad o 
tiempo de residencia del niño, como por la de sus padres. Asimismo, al establecer una residencia legal, excluye a personas que, aun residiendo en el país por un plazo mayor, no pueden computar los años. Vale aclarar que ni el decreto ni la resolución clarifican o distinguen entre tipos de residencia (permanente, temporaria o precaria).

Estas restricciones van en contra de los principios de universalidad, igualdad y no discriminación presentes en la CDN (Art. 14-20), la Ley de Migraciones (Art. 5 y 6), la Ley de Protección Integral de los Derechos de las Niñas, Niños y Adolescentes (Art. 28) y el propio Decreto 1602/09, que presenta explícitamente a la AUH como una medida de carácter universal y basada en el principio del interés superior del niño. "Una política social de carácter universal como la AUH debería actuar como una política de inclusión social de la población migrante, en particular de quienes han migrado en circunstancias de vulnerabilidad y exclusión, las cuales persisten en el país de destino. En este sentido, los objetivos de una política social tan extendida, e incluso novedosa, como la $\mathrm{AUH}$, es precisamente asegurar una cobertura mínima al derecho a la seguridad social de los grupos sociales en situación de exclusión" (Ceriani, Cyment, Morales, 2011, p.5).

La Tarjeta Alimentar (Ley 27.519), que complementa a la AUH, se inserta dentro del Programa Nacional de Seguridad Alimentaria y Nutricional y busca fortalecer el acceso a la canasta básica de alimentos. A pesar de estar fundamentada en el concepto de alimentación como derecho humano, está destinada a madres o padres con hijos e hijas de hasta 6 años de edad o con discapacidad, que reciben la $\mathrm{AUH}$, por lo que, nuevamente, se aplican los requisitos restrictivos frente a la población migrante. 


\section{La respuesta del Estado frente a la emergencia sanitaria COVID-19}

A partir de este contexto restrictivo para el acceso a derechos de manera igualitaria de los niños, niñas y adolescentes migrantes o hijos de migrantes, la emergencia sanitaria COVID-19 — que obligó a repensar las políticas de protección social en general-, se presenta como un escenario nuevo y una oportunidad para, o bien, subsanar los obstáculos que no permiten alcanzar la universalidad de las políticas 0 , por el contrario, profundizar situaciones de discriminación inconstitucionales. La pandemia ha impactado de distintas maneras a la infancia: "[s]i bien la niñez no es el grupo de población más afectado en términos de salud, las niñas y niños son las víctimas ocultas de la pandemia. El COVID-19 no solo puede enfermarlos: también tiene efectos como el aislamiento social, el cierre físico de escuelas y la convivencia en entornos que no siempre son seguros, entre otros. Todas estas situaciones afectan a su educación, los expone a la violencia e impacta en su salud mental” (UNICEF, 2020, p. 3).

Asimismo, el aislamiento social obligatorio — producto de la pandemia - trajo consigo efectos negativos sobre la economía y las fuentes de ingreso de las familias, afectando en mayor medida a aquellas en situación de pobreza, en donde los y las migrantes se encuentran sobrerrepresentados. Para evaluar los efectos de las medidas tomadas por el Estado argentino para hacer frente a esta situación, una vez más se considerará la pobreza monetaria, y no la multidimensional, con el fin de poder analizar los efectos de plazo casi inmediato frente a la emergencia sanitaria. Entre las medidas que el gobierno ha implementado para contener los efectos económicos negativos del confinamiento se encuentran: "los aumentos en los créditos presupuestarios destinados a las políticas alimentarias (\$38 mil millones), los bonos otorgados a perceptores de Asignación Universal por Hijo y Asignación Universal por Embarazo (\$3.100 millones) y la implementación del Ingreso Familiar de Emergencia 
(IFE), con una asignación de $\$ 89.630$ millones" (UNICEF, 2020, p 17). De esta manera, los esfuerzos presupuestarios dirigidos a la protección de ingresos durante la emergencia sanitaria COVID-19 pasaron de $\$ 514$ a $\$ 650$ mil millones de pesos, alcanzando un $2 \%$ del PIB estimado para 2020.

El bono extraordinario de $\$ 3.103$ pesos para los titulares de la AUH también excluye a la población migrante, ya que al estar atada a esta, reproduce los mismos requisitos que la asignación, por lo que es un programa que continúa acentuando las inequidades en el acceso a derechos y va en detrimento de un enfoque de derechos humanos y del principio de universalidad.

El Ingreso Familiar de Emergencia (Decreto 310/2020) es una prestación monetaria no contributiva de carácter excepcional, diseñada específicamente para dar respuesta a la emergencia económica de las familias en mayor situación de vulnerabilidad, en el contexto de la pandemia COVID-19. A pesar de ello, tiene como requisito ser argentino nativo o naturalizado y residente, con una residencia legal en el país no inferior a 2 años. Si bien se reduce la cantidad de años requeridos (aspecto que, por otra parte, sugiere cierta arbitrariedad temporal), una vez más se reproducen parámetros de exclusión, que discriminan no solo a personas migrantes, sino también a niñas, niños y adolescentes argentinos con padres extranjeros. Se coloca el beneficio en cabeza de madres o padres, y no del niño como titular de derecho, exigiéndoles a aquéllos un criterio excluyente que contradice los principios fundamentales de la CDN. Por otra parte, el requisito de residencia legal vuelve a ser ambiguo respecto del tipo de residencia que se requiere y, por último, a estos requisitos se les suma la necesidad de acreditar la situación migratoria regular mediante DNI.

Es importante notar que el decreto explicita, entre sus fundamentos, que "se anticipa que las personas vinculadas al sector informal de la economía, los/las monotributistas de bajos recursos y los trabajadores y las trabajadoras de casas particulares tendrán una 
severa discontinuidad y/o pérdida de sus ingresos durante el período de cuarentena" (Decreto 310/2020) y, dentro de esos grupos, las personas migrantes tienen una presencia elevada. Sin embargo, en lugar de flexibilizar los requisitos de la AUH, la IFE los aumenta, generando aún más obstáculos para su otorgamiento a personas migrantes.

Cabe volver a mencionar que las personas migrantes y refugiadas son quienes han quedado más expuestas ante la emergencia sanitaria COVID-19, debido a la falta de documentación; los altos niveles de informalidad laboral en los que se encuentran; las dificultades habitacionales que enfrentan; el escaso acceso a beneficios $\mathrm{y}$ programas sociales, y a que muchas de ellas realizan trabajos más expuestos al contagio (como las economías de plataforma y servicios de envío, cajeros o vendedores de comercios de alimentos, tareas de cuidado, tareas de limpieza en establecimientos o servicios considerados esenciales, entre otros), los cuales suelen realizarse en condiciones de gran precariedad, informalidad y desprotección. Esta situación se agudiza cuando la migración es reciente, debido a que a la falta de documentación se le suma la escasez de redes familiares y comunitarias de apoyo, las cuales son aún más difíciles de construir durante el periodo de aislamiento social obligatorio.

El cuadro a continuación sintetiza los programas sociales de transferencia de ingresos, tanto en vigencia antes de la emergencia sanitaria COVID-19, como aquéllos diseñados con posterioridad, con el fin de dar cuenta de las diferencias en el acceso a derechos en términos monetarios. 


\begin{tabular}{|c|c|c|c|c|c|}
\hline \multicolumn{2}{|c|}{$\begin{array}{l}\text { Programas previos a la } \\
\text { emergencia COVID-19 }\end{array}$} & \multicolumn{3}{|c|}{$\begin{array}{c}\text { Programas diseñados durante la } \\
\text { emergencia COVID-19 }\end{array}$} & \multirow{2}{*}{$\begin{array}{c}\text { CANASTA } \\
\text { BÀSICA } \\
\text { Junio } 2020\end{array}$} \\
\hline AUH & $\begin{array}{c}\text { Tarjeta } \\
\text { Alimentar }\end{array}$ & $\begin{array}{l}\text { Bono } \\
\text { AUH }\end{array}$ & $\begin{array}{c}\text { Tarjeta } \\
\text { Alimentar } \\
\text { durante } \\
\text { mayo }\end{array}$ & IFE & \\
\hline $\begin{array}{l}\$ 3.293 \text { por } \\
\text { hijo }(\$ 4.241 \\
\text { para } \\
\text { residentes de } \\
\text { determinada } \\
\text { s zonas como } \\
\text { la Provincia } \\
\text { de Buenos } \\
\text { Aires) }\end{array}$ & $\begin{array}{l}\$ 4.000 \text { para } \\
\text { familias con } \\
1 \text { hijo }\end{array}$ & $\$ 3.103$ & $\begin{array}{l}\$ 8.000 \text { para } \\
\text { familias } \\
\text { con } 1 \text { hijo }\end{array}$ & $\$ 10.000$ & $\begin{array}{l}\text { Alimentaria: \$ } \\
5.835\end{array}$ \\
\hline $\begin{array}{l}\$ 10.730 \text { por } \\
\text { hijo con } \\
\text { discapacidad } \\
(\$ 11.159,20 \\
\text { para } \\
\text { residentes de } \\
\text { det. zonas } \\
\text { como la Pcia. } \\
\text { de Buenos } \\
\text { Aires })\end{array}$ & $\begin{array}{l}\$ 6.000 \text { para } \\
\text { familias con } \\
\text { más de un } \\
\text { hijo }\end{array}$ & & $\begin{array}{l}\$ 12.000 \\
\text { para } \\
\text { familias } \\
\text { con más de } \\
\text { un hijo }\end{array}$ & & Total: \$ 14.178 \\
\hline
\end{tabular}

Una familia argentina con dos hijos, ninguno de ellos con discapacidad, que vive en la Ciudad Autónoma de Buenos Aires, recibe mensualmente un total de $\$ 12.586$. Durante los meses de la crisis COVID-19 se adicionaron montos excepcionales, que alcanzan los $\$ 39.103$ (ya que hasta el momento se confirmaron tres pagos del IFE y un pago doble de la Tarjeta Alimentar). Estos montos no han sido otorgados a familias migrantes, que cuentan con altos niveles de informalidad y precariedad laboral, y que presentan niveles de pobreza infantil más altos que el promedio. 


\section{Reflexiones finales}

Es importante reconocer los logros que ha tenido la Argentina en materia normativa, pero es aún más indispensable identificar los desafíos pendientes para generar los cambios necesarios de avance en el cumplimiento de derechos. Las leyes de Protección Integral de los Derechos de Niñas, Niños y Adolescentes y la de Migraciones, así como la AUH y el IFE, tienen como objetivos explícitos contribuir con el desarrollo humano, promover la inclusión social, y hacerlo desde un enfoque de derechos. Para lograrlo, la regularización migratoria, junto con el acceso a derechos en igualdad de condiciones, deben ser garantizados para minimizar las desigualdades y eliminar la pobreza, como se propone en el primero de los Objetivos de Desarrollo Sostenible y la Agenda 2030.

Sin embargo, como se ha evidenciado, los datos de pobreza muestran que, a igual cantidad de miembros, es más probable que un hogar migrante sea pobre en comparación con uno integrado solo por personas nacidas en la Argentina, con una alta incidencia de la pobreza en familias monoparentales o con ambos padres migrantes (tanto jefe o jefa de hogar como cónyuge). Más aun, en el contexto de la emergencia sanitaria COVID-19, con restricciones para la circulación que dificultan el trabajo y hasta han impedido la posibilidad de regreso de muchas personas migrantes, la asistencia del Estado se hace aún más necesaria. Las familias migrantes han visto recortadas o eliminadas sus posibilidades de obtención de ingresos, al estar en gran parte insertas en el mercado laboral informal o cuentapropista, y presentan dificultades habitacionales que entorpecen el cumplimiento de las medidas de distanciamiento social y el acceso a servicios básicos de agua potable y saneamiento. Entre los grupos etarios, los niños, niñas y adolescentes son quienes presentan los índices de pobreza más altos.

En este contexto, la exclusión de niñas, niños y adolescentes solicitantes de la condición de refugiado es particularmente preocupante debido a que, en su mayoría, han sufrido actos de 
violencia física o psicológica, y han debido huir de sus hogares, muchas veces de manera apresurada, sin una planificación previa ni poder traer consigo aquello que pudieran necesitar en los países de acogida. En esta población, la desprotección aumenta dado los tiempos extensos que conllevan los trámites de solicitud de refugio antes la CONARE, durante los cuales las personas cuentan con el Certificado de Residencia Precaria.

En cualquier caso, parece importante recordar la figura del "habitante", prevista en la Constitución Nacional, como portadora de derechos. El Estado tiene el deber de garantizar los derechos de todos los habitantes de su territorio. Este concepto se ha visto reforzado por dos razones: por un lado, porque es el propio Estado quien no permite la libre circulación; por otro, porque en el caso de una pandemia como la del nuevo coronavirus-, si no se cuida a toda la población el contagio crece y se dificulta sustancialmente el control de la enfermedad. En este sentido, se torna imperioso garantizar el acceso a la atención médica básica para las personas migrantes y refugiadas, así como garantizar también el acceso a campañas de inmunización cuando haya disponibilidad de vacunas para el COVID-19.

La existencia de fuertes disparidades entre la población migrante y nativa o naturalizada, requiere dos respuestas clave. En primer lugar, garantizar la universalidad que se declara en la normativa nacional, considerando el acceso al sistema de salud, a la protección social y a cualquier medida adicional de emergencia. En segundo lugar, es necesario implementar medidas adicionales y específicas que complementen las estrategias universales, para mejorar la condición de quienes se encuentran en situaciones de mayor vulnerabilidad, con el fin de no dejar a nadie atrás, como se proclama en la Agenda 2030 de Desarrollo Sostenible. Estas medidas adicionales deben contemplar la especificidad de la población migrante $\mathrm{y}$ refugiada, así como la interseccionalidad que las atraviesa. Las poblaciones no son grupos homogéneos, sino que existen mujeres migrantes, personas migrantes afrodescendientes, personas migrantes con discapacidad, personas refugiadas pertenecientes al 
colectivo LGTBIQ, entre muchas otras. Dado los contextos diferentes, las respuestas para su inclusión deben contemplar esas diferencias. Lo que no debe suceder, claro está, es que las políticas universales los excluyan.

En este sentido, se propone revisar el criterio de dos y tres años de residencia de las medidas, dada su incompatibilidad con la normativa local e internacional, la presencia de requisitos asimétricos con respecto a las asignaciones familiares y, en particular, dada la mayor incidencia de la pobreza en los primeros años de llegada al país. La relación inversa que existe entre la cantidad de años en el país de las personas migrantes y su desprotección no puede ser desoída por quienes diseñan estas políticas públicas. En efecto, la inestabilidad e interrupción de ingresos afecta principalmente a quienes tienen menos de dos años en el país.

En caso de no modificar la cantidad de años requeridos, se propone tomar la residencia de hecho como base para acceder a los programas sociales. Esto cobra importancia frente a la situación que denuncian espacios como el de Agenda Migrante 2020 —en su informe de diciembre de 2019—, sobre un aumento de medidas que han dificultado la regularización migratoria, como el incremento de las tasas a un $1000 \%$ o la puesta en marcha del Ra.D.Ex, un sistema informático complejo de regularización, que dificulta el acceso a las personas migrantes con poca o nula disponibilidad o conocimiento tecnológicos. Asimismo, se propone incorporar a las personas refugiadas, solicitantes de asilo y beneficiarias de programas de visado humanitario, independientemente de la cantidad de años que cuentan en el país.

La emergencia sanitaria COVID-19 está señalando el "elefante en la habitación”, que se encuentra allí hace tiempo. Ha demostrado lo urgente y necesario de contar con un sistema de protección social que esté basado en el principio de universalidad y que tenga la flexibilidad de abarcar diferentes poblaciones vulnerables, contemplando sus características particulares. Frente a estos retos, la Argentina acarrea 
desafíos previos: una Ley de Migraciones que no contempla la especificidad de los derechos de la niñez; una Ley de Protección Integral de los Derechos de Niñas, Niños y Adolescentes carente de una mirada específica sobre las infancias migrantes, y una falta de adecuación normativa de otras leyes, decretos y disposiciones. Los niños y niñas migrantes se encuentran en una situación de doble vulnerabilidad, por su condición de niños y su condición de migrantes, lo que resulta en niveles de pobreza más altos. En el contexto de emergencia sanitaria actual, se profundizan los efectos de las vulneraciones de sus derechos, entre los que se encuentran incrementos aún mayores en los niveles de pobreza, crecientes riesgos para la salud, dificultades para el acceso a la educación debido al cierre de las escuelas, convivencia en entornos que no siempre son seguros y aumento en los niveles de violencia doméstica en contexto de aislamiento. Los cambios no pueden hacerse esperar debido al rápido ritmo de desarrollo de los niños y a la actual gravedad de emergencia sanitaria.

\section{Referencias}

Agenda Migrante 2020 (2020). Informe final sobre la "Situación de la población migrante/extranjera en Argentina ante el avance del Coronavirus", Ciudad de Buenos Aires.

Ceriani, P.; Cyment, P.; Morales, D. (2011). Migración, derechos de la niñez y Asignación Universal por Hijo: las fronteras de la inclusión socialen http://conti.derhuman.jus.gov.ar/2011/10/mesa_2/ceriani_cy ment_morales_mesa_2.pdf (consultado 24 de junio de 2020).

Fondo de las Naciones Unidas para la Infancia (UNICEF), (2020). La pobreza y la desigualdad de niñas, niños y adolescentes en la Argentina. Efectos del COVID-19, Ciudad de Buenos Aires.

Fondo de las Naciones Unidas para la Infancia (UNICEF), Universidad Nacional de Lanús (UNLA), (2013). Niñez, migraciones y derechos humanos en Argentina. Estudio a 10 años de la Ley de Migraciones, Ciudad de Buenos Aires. 
Fondo de las Naciones Unidas para la Infancia (UNICEF), Universidad Nacional de General Sarmiento (UNGS), Instituto de Estudios Laborales y del Desarrollo Económico (IELDE), Equity for children, (2018). Pobreza monetaria y privaciones no monetarias en Argentina, Ciudad de Buenos Aires.

Instituto de Políticas Públicas en Derechos Humanos del MERCOSUR (IPPDH), Organización Internacional para las Migraciones (OIM), 2016. Derechos humanos de la niñez migrante. Disponible en: https://www.ippdh.mercosur.int/wpcontent/uploads/2018/08/IPPDH-MERCOSUR-DerechosHumanos-de-la-Ninez-Migrante.pdf

Instituto Nacional de Estadística y Censos (INDEC), (2020). Condiciones de vida Vol. $4, n^{\circ} 8$. Valorización mensual de la canasta básica alimentaria y de la canasta básica total, Ciudad de Buenos Aires: INDEC.

Organización Internacional de las Migraciones (OIM), Consejo Nacional de Coordinación de Políticas Sociales (CNCPS), (2019). Condiciones de vida de migrantes en la República Argentina, Ciudad de Buenos Aires: OIM.

Organización Internacional de las Migraciones (OIM), Instituto de Políticas Públicas en Derechos Humanos del MERCOSUR (IPPDH), (2016). Derechos humanos de la niñez migrante, Ciudad de Buenos Aires.

Overseas Development Institute (ODI), Fondo de las Naciones Unidas para la Infancia (UNICEF), (2020). Universal child benefits, London: Overseas Development Institute and New York: UNICEF.

Organización de las Naciones Unidas (ONU), 2020. Análisis inicial de las Naciones Unidas COVID-19 en Argentina: impacto socioeconómico y ambiental. Disponible en http://www.onu.org.ar/stuff/Informe-COVID-19-Argentina.pdf (actualizado al 19/06/2020).

Organización de Naciones Unidas, Convención sobre los Derechos del Niño, Nueva York, 1989. Disponible en: https://www.ohchr.org/SP/ProfessionalInterest/Pages/CRC.asp $\underline{\mathrm{X}}$ 
Organización de Naciones Unidas, Convención internacional sobre la protección de los derechos de todos los trabajadores migratorios y de sus familiares, Nueva York, 1990. Disponible en: https://www.ohchr.org/sp/professionalinterest/pages/cmw.asp $\mathrm{X}$

Ley de Migraciones, Ley No 25.871. Disponible en: http://servicios.infoleg.gob.ar/infolegInternet/anexos/9000094999/92016/texact.htm

Ley de Protección Integral de los Derechos de Niñas, Niños y Adolescentes, Ley No 26.061. Disponible en: http://servicios.infoleg.gob.ar/infolegInternet/anexos/110000114999/110778/norma.htm 